\section{Fewer academics could be the answer to insufficient grants}

SIR - The rejection of highquality grant proposals is a problem endemic to universities throughout the world. I suggest that it arises from separating the employment of academics from the central bodies who provide grant funding.

Consider the country of Euphoria. It has just four universities, each of which employs ten academics of comparable quality, and one national funding body. Each academic submits two grants per year, only one of which is rated fundable. So each academic is awarded one grant per year. Into this happy state enters the ambitious new president of the Euphoric University of Fulchester. He makes his mark by doubling the number of academics in his institution. Now Euphoria has 100 grants submitted per year, but still only 40 grants available. Fulchester will get 16 of these and the other universities will now get only eight each.

This is a rational action by the new president, as the rewards from obtaining six additional grants are so great that it is worth hiring the 10 new staff. Euphoria loses overall, however, because its taxpayers and students are now paying to employ 10 extra staff, with the same amount of research being done. The other four universities also lose, as they are now receiving two fewer grants. The incentive will be for them to act in a similar way until Euphoria stabilizes, with many more excellent grants being submitted than can be funded.

This situation naturally arises in an environment in which employing academic staff is separated from obtaining research funding. We would be better off having fewer academics and using the savings to fund more grants, because then more research could be done for the same national expenditure. Such action has to be taken by governments, as universities currently have the freedom to over-staff and are rewarded for doing so under the present system.

Andrew Doig Manchester Interdisciplinary Biocentre, University of Manchester, 131 Princess Street, Manchester M17DN, UK

\section{Working together to put molecules on the map}

SIR - We applaud the call in your Editorial 'A place for everything'

(Nature 453, 2; 2008) for researchers to record the latitude and longitude of their data, in order to place all biological samples in proper spatial (and temporal) context. We agree that this minimum information guideline should apply to all biological samples taken from the natural environment, and note the pressing need for relevant molecular data to be tagged with geographical location.

The International Nucleotide Sequence Database Collaboration - comprising the DNA Data Bank of Japan, the European Molecular Biology Laboratory and GenBank - already offers the option of recording latitude and longitude coordinates. This qualifier, among others, was requested by the Consortium for the Barcode of Life to provide the geographical origin of molecules it uses to identify organisms. The "minimum information about a genome sequence" guideline published by the Genomic Standards

Consortium (Nature Biotech. 26, 541-547; 2008) calls for this critical field to be mandatory for all genome and metagenome submissions, along with altitude or depth and time of sampling.

Other molecules that are equally critical to tag with this information are the vast number of other marker genes, especially $16 \mathrm{~S}$ and $18 \mathrm{~S}$ ribosomal RNA sequences, that are being generated globally from a diverse

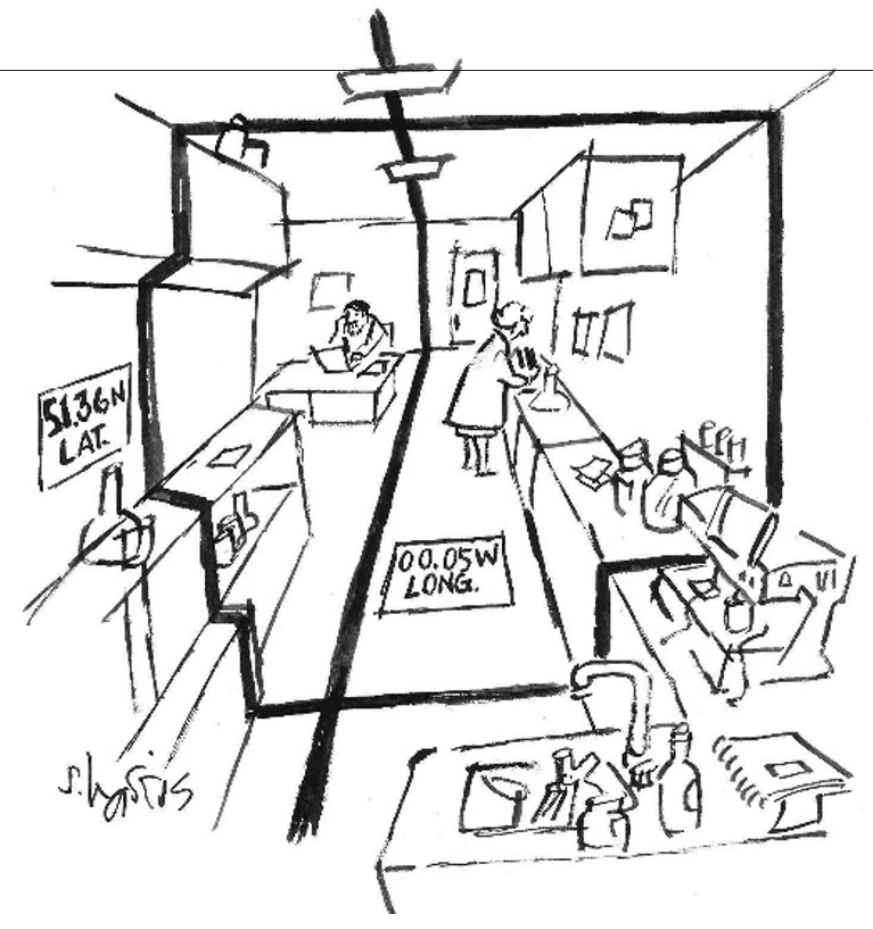

range of habitats. This registration becomes all the more relevant as ultra-high-throughput sequencing of these molecules continues to be more widely applied. Core to these efforts are projects such as the Environment Ontology and Gazetteer initiatives, which describe environments and place names, respectively. Combined, these resources will support the consistent annotation and retrieval of environmental information associated with an organism or biological sample.

These projects all highlight the growing importance of community-driven initiatives in developing improved standards for reporting experimental data. We look forward to the day when it will be commonplace to view collections of molecules 'on the map', so to speak, such that questions relating to their global and local abundances, distributions, environments and functions can be properly addressed. Getting to this point will require: increased awareness; higher expectations for the quality and quantity of descriptive data recorded; improved standards, ontologies and databases; proof of the value of downstream analyses; and widespread practical changes, such as use of hand-held devices for recording real-time contextual information (and, in the future, for generating data) in the field.

Dawn Field NERC Centre for Ecology and Hydrology, Oxford OX13SR, UK This letter was also signed by the following, whose addresses can be found at http://gensc.org: Norman Morrison, Frank Oliver Glöckner, Renzo Kottmann, Guy Cochrane, Robert Vaughan, George Garrity, Jim Cole, Lynette Hirschman, Lynn Schriml, Ilene Mizrachi, Scott Federhen, David Schindel, Scott Miller, Paul Hebert, Sujeevan Ratnasingham, Robert Hanner, Linda Amaral-Zettler, Mitchell Sogin, Michael Ashburner, Suzanna Lewis, Barry Smith, Genomic Standards Consortium (GSC), International Nucleotide Sequence Database Collaboration (INSDC), Consortium for the Barcode of Life (CBOL), International Census of Marine Microbes (ICoMM), Environment Ontology Consortium (EnvO)

\section{Decoherence does not get rid of the quantum paradox}

SIR - In his Essay 'Lifting the fog from the north' (Nature 453, 39; 2008), Maximilian Schlosshauer describes how the process of 


\section{"The undeciphered Phaistos Disc is perhaps the most infamous of ancient inscriptions." Andrew Robinson, page 990}

decoherence can explain the famous double-slit experiment. An electron interacting with innumerable quanta in the photographic plate (and its environment) becomes entangled with all of them - and the resulting collective wavefunction is so narrow that it appears particle-like.

But the question remains as to why the wavefunction narrows in precisely the location where it does, or - as Schlosshauer puts it - "Why is a single spot here and not there?"

The author's somewhat 'foggy' answer is suggestive of a version of Everett's 'many worlds' idea (see Nature 448, 15-17; 2007), in which all possible branches of the wavefunction continue to exist autonomously. But this interpretation merely shifts the question to "Why do I find myself experiencing the branch/world with the spot here and not the branch/world with the spot there?"

We still have no answer and if there is one, decoherence is at best only part of it (S. L. Adler Stud. Hist. Philos. Sci. 34, 135-142; 2003). As Joos and Zeh remarked on decoherence as a source of spatial localization: "Of course no unitary treatment of the time dependence can explain why only one of these dynamically independent components is experienced." (E. Joos and H. D. Zeh Zeitschrift Phys. B 59, 223-243; 1985).

We are still left with a dichotomy: on the one hand, infinitely many continuously distributed potentialities, and on the other, one narrow, irreversibly realized actuality. Contrary to Schlosshauer's conclusions, complementary (mutually incompatible) descriptions are necessary to describe the landscape we are currently experiencing, even as the fog is lifting.

Nikolaus von Stillfried Department of Environmental Health Science, University of Freiburg, Breisacherstrasse 115B, 79106 Freiburg, Germany

\section{Ventures should not overstate their aims just to secure funding}

be used as a reason to limit adaptation to climate change. Bring on the revolution if you will, but don't mistake it for Utopia.

Mike Hulme, Suraje Dessai Tyndall Centre, School of Environmental Sciences, University of East Anglia, Norwich NR4 7TJ, UK

conceived with the best intentions, but so easily claim more than is plausible and more than can ever be delivered. We fear that the "revolution in climate prediction" called for by the World Modelling Summit for Climate Prediction and reported in your journal ('They say they want a revolution' Nature $\mathbf{4 5 3}$, 268-269; 2008) will fall foul of the same hubris.

Any venture bidding for investment that exceeds a billion dollars needs to have wellgrounded justifications. Advancing our basic understanding of how the climate system works through enhanced representation of that system in next-generation climate models - ("pure intellectual excitement") - may indeed be such justification. But claiming that this will allow scientists to "provide answers to key questions ... such as future food supply" and guide decisions the world will be making to cope with climate change displays a misunderstanding of the nature of adaptation and its contingency on our imagining of future social change.

The reason that the UK summit at Reading University overclaimed the benefits of climate prediction for adaptation in its pitch for a billion dollars of new science investment is revealed by the summit's chair, Jagadish Shukla, in his warning: "If we just ask for enhanced understanding, then we have very little chance of getting the necessary funding".

Effective and robust adaptation strategies are not significantly limited by the absence of accurate and precise regional climate predictions. They are limited more by a multitude of technological, institutional, cultural, economic and psychological factors that lie beyond the reach of climate models - and always will. The epistemological limits to predicting future climates with accuracy and precision must not

\section{Digital identifiers work for articles, so why not for authors?}

SIR - Several Correspondences, including 'Give south Indian authors their true names' (Nature 452, 530; 2008) and 'Name variations can hit citation rankings' (Nature 453, 450; 2008), have illustrated difficulties in identifying authors and their papers, citations and $h$-index

In an academic world in which decisions on promotion and funding often depend on the applicant's scientific impact, an incorrect publication or citation record in an online database can be very inconvenient. Scopus and Thomson's Web of Science, which make available abstract and citation databases, acknowledge the issue and have come up with solutions: the Author Identifier and ResearcherID, respectively.

These systems assign an identifying code to each author. Unfortunately, a single author can have more than one Author Identifier in Scopus (I am cryptically known as 7006716603 and 16551750300). And as only invited researchers can register for a number, ResearcherID is not yet used as a unique author key in the Web of Science - making it difficult to differentiate me from a highly cited ecologist from the Netherlands, despite the 'Distinct Author Sets' feature.

If it is possible to have DOls for objects (or, so they say, enough IPv6 addresses for every molecule on Earth), why is it so difficult to implement DAls for authors?

Raf Aerts Division Forest, Nature and Landscape, Katholieke Universiteit Leuven, Celestijnenlaan 200E-2411, 3001 Leuven, Belgium

\section{Europe needs to protect its transgenic crop research}

SIR - On 5 June 2008, our authorized, small-scale field trial of transgenic potato plants for nematode control was destroyed by people seeking to coerce government and society. It was one of only two trials authorized in the United Kingdom this year.

Our concern is that Directive 2001/18/EC, the European Union (EU) legislation that governs such trials, is confused. Although it recognizes the need for field releases at the research stage (clause 23), it does not distinguish between these and developmentstage trials in its risk assessments. It has also set the legal precedent of providing precise locations of trial sites to vandals.

We have no evidence that the 400 transgenic plants we released posed any environmental concern, particularly when considered in the context of the annual UK potato crop of 8,000 million plants and their naturally hazardous glycoalkaloid content.

If EU governments cannot protect the trials they authorize, they should establish secure, vandal-proof national testing centres.

Unfortunately, a failure to distinguish a research trial from product-development trials seems to have blinded activists to the published, broader aims of our work. We develop controls for nematodes on subsistence crops in Africa and Asia, where both farmers and governments recognize the need for new technologies.

What is the distinction between burning university books 75 years ago and now destroying university research intended for publication in scientific journals? European governments must ensure that science in our universities can progress without coercion.

Howard J. Atkinson, Peter E. Urwin Centre for Plant Sciences, University of Leeds, Leeds LS2 9JT, UK 